could cause heightened cortical excitability and hence unilateral hallucinations.

(g) Hallucinations can sometimes lateralise to the good ear (Robinson, 1927). Electrical aural stimulation in cases of hallucinations and ear disease paradoxically affected the opposite ear (Ireland, 1893). In McCay \& Healey's (1931) case a right radical mastoidectomy cured long-standing mental disorder including sinistral hallucinations. No brain disease was detected. A similar case of 'toxic insanity' came to post-mortem (Henderson et al, 1913). A man became very confused and irritable with hallucinations during an acute exacerbation of chronic otitis. There was considerable improvement of his mental condition with successful ear treatment, but he relapsed and died. There were old and new labyrinthine bone fistulas, but the brain was remarkably healthy.

(h) 'Temporal lobe' epilepsy can also arise from the ear (Ireland, 1893).

(i) Hallucinations can be attenuated by plugging the ear or induced by removing impacted wax (Ireland, 1893).

(j) Peripheral audiosensitivity can lead into hallucinations (Ireland, 1893).

\section{Love Walk}

A. G. GordoN

London SE5 8AD

\section{References}

GoRDon, A. G. (1987) Otopathic hallucinations. Acta Psychiatrica Scandinavica, 75, 664-668.

Henderson, D. K., Mutrhead, W. \& Fraser, J. S. (1913) A case of toxic exhaustive insanity associated with chronic suppurative otitis media, labyrinthitis, and extra-dural abscess. Review of Neurology and Psychiatry, 11, 565-576.

IRELAND, W. W. (1893) German retrospect. Journal of Mental Disease, 39, 299-302.

MCCAY, A. \& Healey, F. H. (1931) Aural sepsis in relation to mental disorder. Journal of Mental Disease, 77, 193-195.

RoBnson, G. W. (1927) Aural disease in the insane. Journal of Neurology and Psychopathology, 7, 332-337.

SIR: We were interested to read the letter of Khan et al (Journal, February 1988, 152, 297-298) concerning a patient with unilateral complex auditory hallucinations. We report two further cases.

Case Reports: (i) A 58-year-old man was readmitted with his third episode of psychotic depression, characterised by depressed mood, loss of drive, grossly disturbed sleep, and loss of appetite and weight. Mental state examination revealed complaints of "a whistling noise", louder in his left ear, which he attributed to a "surveillance device", and derogatory "whispering voices" present only on the left side. During a previous admission the whistling had been accompanied by a single male voice As his mood improved, the voices faded and disappeared, leaving only a distant whistling in his left ear. Physical examination showed a marked sensorineural deafness in his left ear. He failed to attend for audiometry after discharge. All other investigations were normal.

(ii) A 47-year-old woman had initially been seen by psychiatrists at the age of 18 years because of a sensitive, inadequate personality. Alcohol dependence developed, complicated by grand mal epilepsy. She first complained of auditory hallucinations at the age of 33 years. During subsequent admissions, passivity phenomena, thought insertion, and broadcasting were documented at various times. Mental state examination during the most recent admission revealed a woman of low average intelligence, with slight blunting of affect. She described a persistent buzzing and a solitary, adolescent, female voice, both audible only in her left ear. Four EEGs over a period of 21 years showed diffuse slowing and bilateral spike activity with photic stimulation, most marked over the temporal leads. Audiometry showed bilateral high tone deafness; other investigations were normal.

In both these cases, unilateral hallucinations were present with hearing loss and ipsilateral tinnitus. In the first case the phenomenon appears to represent auditory hyperaesthesia during a depressive episode, leading to functional hallucinations (Hamilton, 1974). In the second case there was additional evidence of non-focal epileptiform activity which may have contributed to both the psychosis and the hallucinations.

ANDREW MCBRIDE
LAWRENCE HAMILTON-KIRKWOOD
$\begin{aligned} & \text { St Cadoc's Hospital } \\ & \text { Caerleon } \\ & \text { Gwent }\end{aligned}$
Reference
HAmitron, M. (1974) Fish's Clinical Psychopathology. Bristol:
Wright.

\section{Inadequate Seizures with Electroconvulsive Therapy}

SIR: Sharpe \& Andrew recently published a Brief Report (Journal, January 1988, 152, 134-136) citing the case of a severely depressed patient in whom treatment with electroconvulsive therapy was abandoned because of repeated failure to induce a convulsion. The authors suggested that failure to convulse may be an occasional and unrecognised cause of non-response to ECT, and concluded that adequate monitoring of seizure activity is required. 